\title{
RIGHLIGHTS
}

IBD

\section{Modified Lactobacillus acidophilus may regulate intestinal inflammation}

Lactobacillus acidophilus that lack

lipoteichoic acid (a major component of the cell walls of Gram-positive bacteria) have an anti-inflammatory effect in mice that results in resistance to colitis, according to a US research group.

"The gastrointestinal microbiota and the immune system are closely tied, and constantly influence each other," explains lead author Mansour Mohamadzadeh from Northwestern University, Chicago, IL. "However, the cellular and molecular mechanisms by which intestinal commensals and their bacterial products impact autoimmune responses at distal sites remain enigmatic."

To investigate these mechanisms and the importance of lipoteichoic acid in colitis, the authors deleted the gene that encodes the phosphoglycerol transferase that synthesizes the glycerol chain of lipoteichoic acid in the NCFM strain of L. acidophilus.
L. acidophilus deficient in lipoteichoic acid induced the expression of regulatory signals (such as interleukin 10) and downregulated co-stimulatory molecules (such as CD40) in mouse dendritic cells. Mice treated with L. acidophilus that did not express lipoteichoic acid showed regulatory immunity and were resistant to colitis induced by dextran sulfate sodium or T cells. In mice with established colitis, treatment with L. acidophilus that lacked lipoteichoic acid resulted in improvements in their colitis symptoms.

L. acidophilus NCFM is widely regarded as safe for human consumption and has been included in a range of human foods (such as yogurts and probiotic supplements) since the 1970s. These bacteria could, therefore, be used as oral delivery vehicles for vaccines and therapeutics. In particular, the findings from this study could have implications for the treatment of patients with IBD.

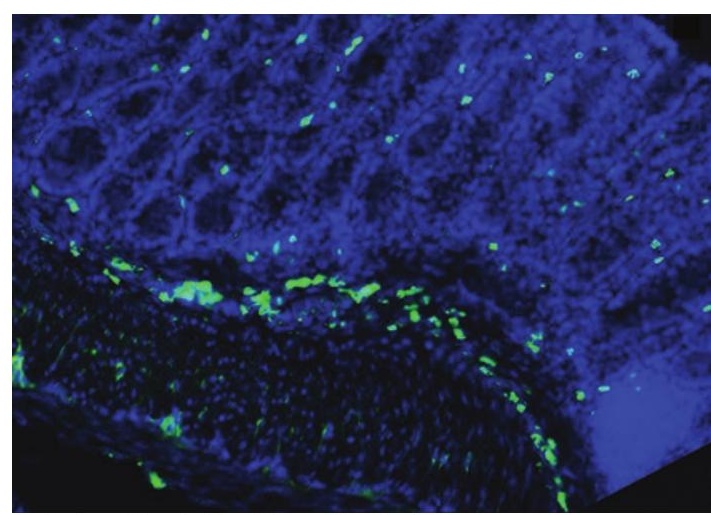

Production of interleukin 10 (green) in colonic mucosa of mice treated with Lactobacillus acidophilus deficient in lipoteichoic acid. Courtesy of M. Mohamadzadeh.

"We now hope to evaluate the antiinflammatory effects of this probiotic culture in a variety of animal and human studies to determine efficacy for treatment, prevention of intestinal inflammation ... and colon cancer," concludes Mohamadzadeh.

Claire Greenhill

Original article Mohamadzadeh, M. et al. Regulation of induced colonic inflammation by Lactobacillus acidophilus deficient lipoteichoic acid. Proc. Natl Acad. Sci. USA doi:10.1073/pnas.1005066107 